\title{
Application of Homotopy Perturbation and Variational Iteration Methods for Fredholm Integrodifferential Equation of Fractional Order
}

\author{
Asma Ali Elbeleze, ${ }^{1}$ Adem Kiliçman, ${ }^{2}$ and Bachok M. Taib ${ }^{1}$ \\ ${ }^{1}$ Faculty of Science and Technology, Universiti Sains Islam Malaysia, 71800 Nilai, Malaysia \\ ${ }^{2}$ Department of Mathematics and Institute for Mathematical Research, University Putra Malaysia, \\ 43400 UPM, Serdang, Selangor, Malaysia \\ Correspondence should be addressed to Adem Kiliçman, akilicman@putra.upm.edu.my
}

Received 20 May 2012; Revised 4 September 2012; Accepted 4 September 2012

Academic Editor: Dumitru Bǎleanu

Copyright (C) 2012 Asma Ali Elbeleze et al. This is an open access article distributed under the Creative Commons Attribution License, which permits unrestricted use, distribution, and reproduction in any medium, provided the original work is properly cited.

This paper presents the application of homotopy perturbation and variational iteration methods as numerical methods for Fredholm integrodifferential equation of fractional order with initialboundary conditions. The fractional derivatives are described in Caputo sense. Some illustrative examples are presented.

\section{Introduction}

Fractional differential equations have attracted much attention, recently, see for instance [1-4]. This is mostly due to the fact that fractional calculus provides an efficient and excellent instrument for the description of many practical dynamical phenomena arising in engineering and scientific disciplines such as, physics, chemistry, biology, economy, viscoelasticity, electrochemistry, electromagnetic, control, porous media and many more, see for example, $[5,6]$.

During the past decades, the topic of fractional calculus has attracted many scientists and researchers due to its applications in many areas, see [4, 7-9]. Thus several researchers have investigated existence results for solutions to fractional differential equations, see $[10,11]$. Further, many mathematical formulation of physical phenomena lead to integrodifferential equations, for example, mostly these type of equations arise in fluid dynamics, biological models and chemical kinetics, and continuum and statistical mechanics, for more details see [12-16]. Integrodifferential equations are usually difficult to 
solve analytically, so it is required to obtain an efficient approximate solution. The homotopy perturbation method and variational iteration method which are proposed by $\mathrm{He}$ [17-26] are of the methods which have received much concern. These methods have been successfully applied by many authors, such as the works in $[19,27,28]$.

In this work, we study the Integrodifferential equations which are combination of differential and Fredholm-Volterra equations that have the fractional order. In particular, we applied the HPM and VIM for fractional Fredholm Integrodifferential equations with constant coefficients

$$
\sum_{k=0}^{\infty} P_{k} D_{*}^{\alpha} y(t)=g(t)+\lambda \int_{0}^{a} H(x, t) y(t) d t, \quad a \leq x, t \leq b,
$$

under the initial-boundary conditions

$$
\begin{aligned}
& D_{*}^{\alpha} y(a)=y(0), \\
& D_{*}^{\alpha} y(0)=y^{\prime}(a),
\end{aligned}
$$

where $a$ is constant, and $1<\alpha<2$, and $D_{*}^{\alpha}$ is the fractional derivative in the Caputo sense.

For the geometrical applications and physical understanding of the fractional Integrodifferential equations, see [14, 26]. Further, we also note that fractional integrodifferential equations were associated with a certain class of phase angles and suggested a new way for understanding of Riemann's conjecture, see [29].

In present paper, we apply the HPM and VIM to solve the linear and nonlinear fractional Fredholm Integrodifferential equations of the form (1.1). The paper is organized as follows. In Section 2, some basic definitions and properties of fractional calculus theory are given. In Section 3, the basic idea of HPM exists. In Section 4, also is the basic idea of VIM. In Sections 5 and 6, analysis of HPM and VIM exsists, respectively. some examples are given in Section 7. Concluding remarks are listed in Section 8.

\section{Preliminaries}

In order to modeling the real world application the fractional differential equations are considered by using the fractional derivatives. Thus, in this section, we give some basic definitions and properties of fractional calculus theory which is used in this paper. There are many different starting points for the discussion of classical fractional calculus, see for example, [30]. One can begin with a generalization of repeated integration. If $f(t)$ is absolutely integrable on $[0, b)$, as in [31] then

$$
\int_{0}^{t} d t_{n} \int_{0}^{t_{n}} d t_{n-1} \cdots \int_{0}^{t_{3}} d t_{2} \int_{0}^{t_{2}} f\left(t_{1}\right) d t_{1}=\frac{1}{(n+1) !} \int_{0}^{t}\left(t-t_{1}\right)^{n-1} f\left(t_{1}\right) d t_{1}=\frac{1}{(n+1) !} t^{n-1} * f(t),
$$


where $n=1,2, \ldots$, and $0 \leq t \leq b$. On writing $\Gamma(n)=(n-1) !$, an immediate generalization in the form of the operation $I^{\alpha}$ defined for $\alpha>0$ is

$$
\left(I^{\alpha} f\right)(t)=\frac{1}{\Gamma(\alpha)} \int_{0}^{t}\left(t-t_{1}\right)^{\alpha-1} f\left(t_{1}\right) d t_{1}=\frac{1}{\Gamma(\alpha)} t^{\alpha-1} * f(t), \quad 0 \leq t<b,
$$

where $\Gamma(\alpha)$ is the Gamma function and $t^{\alpha-1} * f(t)=\int_{0}^{t} f\left(t-t_{1}\right)^{\alpha-1}\left(t_{1}\right) d t_{1}$ is called the convolution product of $t^{\alpha-1}$ and $f(t)$. Equation (2.2) is called the Riemann-Liouville fractional integral of order $\alpha$ for the function $f(t)$. Then, we have the following definitions.

Definition 2.1. A real function $f(x), x>0$ is said to be in space $C \mu, \mu \in \mathbb{R}$ if there exists a real number $p>\mu$, such that $f(x)=x^{p} f_{1}(x)$, where $f_{1}(x) \in C(0, \infty)$, and it is said to be in the space $C_{\mu}^{n}$ if $f^{n} \in \mathbb{R}_{\mu}, n \in \mathbb{N}$.

Definition 2.2. The Riemann-Liouville fractional integral operator of order $\alpha \geq 0$ of a function $f \in C \mu, \mu \geq-1$ is defined as

$$
J^{\alpha} f(x)=\frac{1}{\Gamma(\alpha)} \int_{0}^{x}(x-t)^{\alpha-1} f(t) d t, \quad \alpha>0, t>0
$$

In particular, $J^{0} f(x)=f(x)$.

For $\beta \geq 0$ and $\gamma \geq-1$, some properties of the operator $J^{\alpha}$ :

(1) $J^{\alpha} J^{\beta} f(x)=J^{\alpha+\beta} f(x)$,

(2) $J^{\alpha} J^{\beta} f(x)=J^{\beta} J^{\alpha} f(x)$,

(3) $J^{\alpha} x^{\gamma}=(\Gamma(\gamma+1) / \Gamma(\alpha+\gamma+1)) x^{\alpha+\gamma}$.

Definition 2.3. The Caputo fractional derivative of $f \in C_{-1}^{m}, m \in \mathbb{N}$ is defined as

$$
D^{\alpha} f(x)=\frac{1}{\Gamma(m-\alpha)} \int_{0}^{x} f^{m}(t) d t, \quad m-1<\alpha \leq m
$$

Lemma 2.4. If $m-1<\alpha \leq m, m \in \mathbb{N}, f \in C_{\mu}^{m}, \mu>-1$ then the following two properties hold

(1) $D^{\alpha}\left[J^{\alpha} f(x)\right]=f(x)$,

(2) $J^{\alpha}\left[D^{\alpha} f(x)\right]=f(x)-\sum_{k=1}^{m-1} f^{k}(0)\left(x^{k} / k !\right)$.

Now, if $f(x)$ is expanded to the block pulse functions, then the Riemann-Liouville fractional integral becomes

$$
\left(I^{\alpha} f\right)(x)=\frac{1}{\Gamma(\alpha)} x^{\alpha-1} * f(x) \simeq \xi^{T} \frac{1}{\Gamma(\alpha)}\left\{x^{\alpha-1} * \phi_{m}(x)\right\}
$$

Thus, if $x^{\alpha-1} * \phi_{m}(x)$ can be integrated, then expanded in block pulse functions, the RiemannLiouville fractional integral is solved via the block pulse functions. Thus, one notes on that 
Kronecker convolution product can be expanded in order to define the Riemann-Liouville fractional integrals for matrices by using the Block Pulse operational matrix as follows:

$$
\frac{1}{\Gamma(\alpha)} \int_{0}^{t}\left(t-t_{1}\right)^{\alpha-1} \phi_{m}\left(t_{1}\right) d t_{1} \simeq F_{\alpha} \phi_{m}(t)
$$

where

$$
F_{\alpha}=\left(\frac{b}{m}\right)^{\alpha} \frac{1}{\Gamma(\alpha+2)}\left[\begin{array}{ccccc}
1 & \xi_{2} & \xi_{3} & \cdots & \xi_{m} \\
0 & 1 & \xi_{2} & \cdots & \xi_{m-1} \\
0 & 0 & 1 & \cdots & \xi_{m-2} \\
0 & 0 & 0 & \ddots & \vdots \\
0 & 0 & 0 & 0 & 1
\end{array}\right]
$$

see [32].

\section{Homotopy Perturbation Method}

To illustrate the basic idea of this method, we consider the following nonlinear differential equation:

$$
A(u)-f(r)=0, \quad r \in \Omega,
$$

with boundary conditions

$$
B\left(u, \frac{\partial u}{\partial n}\right)=0, \quad r \in \Gamma
$$

where $A$ is a general differential operator; $B$ is a boundary operator; $f(r)$ is a known analytic function, and $\Gamma$ is the boundary of the domain $\Omega$.

In general, the operator $A$ can be divided into two parts $L$ and $N$, where $L$ is linear, while $N$ is nonlinear. Equation (3.1) therefor, can be rewritten as follows:

$$
L(u)+N(u)-f(r)=0 .
$$

By the homotopy technique [33-35], we construct a homotopy $v(r, p): \Omega \times[0,1] \rightarrow \mathbb{R}$ which satisfies

$$
H(v, p)=(1-p)\left[L(v)-L\left(u_{0}\right)\right]+p[A(v)-f(r)]=0, \quad p \in[0,1], r \in \Omega
$$

or

$$
H(v, p)=L(v)-L\left(u_{0}\right)+p L\left(u_{0}\right)+p[N(v)-f(r)]=0,
$$


where $p \in[0,1]$ is an embedding parameter, and $u_{0}$ is an initial approximation of (3.1) which satisfies the boundary conditions. From (3.2) and (3.3) we have

$$
\begin{gathered}
H(v, 0)=L(v)-L\left(u_{0}\right)=0, \\
H(v, 1)=A(v)-f(r)=0
\end{gathered}
$$

the changing in the process of $p$ from zero to unity is just that of $v(r, p)$ from $u_{0}(r)$ to $u(r)$. In topology, this called deformation, and $L(v)-L\left(u_{0}\right)$ and $A(v)-f(r)$ are called homotopic. Now, assume that the solution of (3.2) and (3.3) can be expressed as

$$
v=v_{0}+p v_{1}+p^{2} v_{2}+\cdots
$$

Setting $p=1$ results in the approximate solution of (3.1).

Therefore,

$$
u=\lim _{p \rightarrow 1} v=v_{0}+v_{1}+v_{2}+\cdots
$$

\section{The Variational Iteration Method}

To illustrate the basic concepts of VIM, we consider the following differential equation

$$
L(u)+N(u)=g(x),
$$

where $L$ is a linear operator; $N$ is nonlinear operator, and $g(x)$ is an nonhomogeneous term. According to VIM, one constructs a correction functional as follows:

$$
y_{n+1}=y_{n}+\int_{0}^{x} \lambda\left[L y_{n}(s)-N \widetilde{y_{n}}(s)\right] d s,
$$

where $\lambda$ is a general Lagrange multiplier, and $\widetilde{y_{n}}$ denotes restricted variation that is $\delta \widetilde{y_{n}}=0$.

\section{Analysis of Homotopy Perturbation Method}

To illustrate the basic concepts of HPM for Fredholm Integrodifferential equation (1.1) with boundary conditions (1.2) and (1.3). We use the view of He in $[19,20]$, where the following homotopy was constructed for (1.1) as the following:

$$
(1-p) \sum_{k=0}^{\infty} P_{k} D_{*}{ }^{\alpha} y(x)+p\left[\sum_{k=0}^{\infty} P_{k} D_{*}{ }^{\alpha} y(x)-g(t)-\lambda \int_{a}^{b} H(x, t) y(x) d x\right]=0
$$

or

$$
\sum_{k=0}^{\infty} P_{k} D_{*}{ }^{\alpha} y(x)=p\left[g(t)+\lambda \int_{a}^{b} H(x, t) y(x) d x\right]
$$


where $p \in[0,1]$ is an embedding parameter. If $p=0$, (5.2) becomes linear fractional differential equation

$$
\sum_{k=0}^{\infty} P_{k} D_{*}^{\alpha} y(x)=0
$$

and when $p=1$, the (5.2) turn out to be the original equation. In view of basic assumption of HPM, solution of (1.1) can be expressed as a power series in $p$

$$
y(x)=y_{0}(x)+p_{1} y_{1}(x)+p_{2} y_{2}(x)+\cdots
$$

when $p=1$, we get the approximate solution of (5.4)

$$
y(x)=y_{0}(x)+y_{1}(x)+y_{2}(x)+\cdots
$$

The convergence of series (5.5) has been proved in [21]. Substitution (5.4) into (5.2), and equating the terms with having identical power of $p$, we obtain the following series of equations:

$$
\begin{aligned}
& p^{0}: \sum_{k=0}^{\infty} P_{k} D_{*}^{\alpha} y_{0}=0, \\
& p^{1}: \sum_{k=0}^{\infty} P_{k} D_{*}^{\alpha} y_{1}=g(t)-\lambda \int_{a}^{b} H(x, t) y_{0}(x) d x, \\
& p^{2}: \sum_{k=0}^{\infty} P_{k} D_{*}^{\alpha} y_{2}=-\lambda \int_{a}^{b} H(x, t) y_{1}(x) d x, \\
& p^{3}: \sum_{k=0}^{\infty} P_{k} D_{*}^{\alpha} y_{3}=-\lambda \int_{a}^{b} H(x, t) y_{2}(x) d x, \\
& \quad:
\end{aligned}
$$

with the initial-boundary conditions

$$
D_{*}^{\alpha} y(a)=y(0), \quad D_{*}^{\alpha} y(0)=y^{\prime}(a)
$$

The initial approximation can be chosen in the following manner.

$$
y_{0}=\sum_{j=0}^{1} \gamma_{j} \frac{x^{j}}{j !}=\gamma_{0}+\gamma_{1} x, \quad \text { where } \gamma_{0}=D_{*}^{\alpha} y(a) \gamma_{1}=D_{*}^{\alpha} y(0)
$$


Note that the (5.6) can be solved by applying the operator $J_{*}^{\alpha}$ and by some computation, we approximate the series solution of HPM by the following $N$-term truncated series

$$
x_{n}(x)=y_{0}(x)+y_{1}(x)+\cdots+y_{N-1}(x)
$$

which is the approximate solution of (1.1)-(1.3).

\section{Analysis of VIM}

To solve the fractional Integrodifferential equation by using the variational iteration method, with boundary conditions (1.2) and (1.3) we construct the following correction functional:

$$
y_{k+1}(x)=y_{k}(x)+J^{\alpha}\left[\mu\left(\sum_{k=0}^{\infty} P_{k} D_{*}^{\alpha} y(x)-\tilde{g}(x)-\lambda \int_{0}^{a} H(x, s) \tilde{y}_{k}(s) d s\right)\right]
$$

or

$$
y_{k+1}(x)=y_{k}(x)+\frac{1}{\Gamma(\alpha)} \int_{0}^{x}(x-s)^{\alpha-1} \mu(s)\left[\sum_{k=0}^{\infty} P_{k} D_{*}^{\alpha} y(s)-\tilde{g}_{k}(s)-\lambda \int_{0}^{a} H(x, p) \tilde{y}_{k}(p) d p\right]
$$

where $\mu$ is a general Lagrange multiplier, and $\tilde{g}_{k}(x)$ and $\tilde{y}_{k}(x)$ are considered as restricted variation, that is, $\delta \widetilde{g}_{k}(x)=0$ and $\delta \tilde{y}_{k}(x)=0$.

Making the above correction functional stationary, the following condition can be obtained

$$
\delta y_{k+1}(x)=\delta y_{k}(x)+\int_{0}^{x}(x-s)^{\alpha-1} \mu(s)\left[\sum_{k=0}^{\infty} P_{k} \delta D_{*}^{\alpha} y(s)-\delta \tilde{g}_{k}(s)-\lambda \int_{0}^{a} H(x, p) \delta \tilde{y}_{k}(p) d p\right] .
$$

It's boundary condition can be obtained as follows:

$$
1-\left.\mu^{\prime}(s)\right|_{x=s}=0,\left.\quad \mu(s)\right|_{x=s}=1 .
$$

The Lagrange multipliers can be identified as follows:

$$
\mu(s)=\frac{1}{2}(x-s) .
$$


We obtain the following iteration formula by substitution of (6.5) in (6.2):

$$
\begin{aligned}
& y_{k+1}(x) \\
& \quad=y_{k}(x)+\frac{1}{2 \Gamma(\alpha-1)} \int_{0}^{x}(x-s)^{\alpha-2}(s-x)\left[\sum_{k=0}^{\infty} P_{k} D_{*}^{\alpha} y(s)-\tilde{g}_{k}(s)-\lambda \int_{0}^{a} H(x, p) \tilde{y}_{k}(p) d p\right] d s .
\end{aligned}
$$

That is,

$$
y_{k+1}(x)=y_{k}(x)-\frac{(\alpha-1)}{2 \Gamma(\alpha)} \int_{0}^{x}(x-s)^{\alpha-1}\left[\sum_{k=0}^{\infty} P_{k} D_{*}^{\alpha} y(s)-\tilde{g}_{k}(s)-\lambda \int_{0}^{a} H(x, p) \tilde{y}_{k}(p) d p\right] d s
$$

This yields the following iteration formula:

$$
y_{k+1}(x)=y_{k}(x)-\frac{(\alpha-1)}{2} J^{\alpha}\left[\sum_{k=0}^{\infty} P_{k} D_{*}^{\alpha} y(x)-g_{k}(x)-\lambda \int_{0}^{a} H(x, s) \tilde{y}_{k}(s) d s\right] .
$$

The initial approximation $y_{0}$ can be chosen by the following manner which satisfies initialboundary conditions (1.2)-(1.3)

$$
y_{0}=\gamma_{0}+\gamma_{1} x, \quad \text { where } \gamma_{0}=D_{*}^{\alpha} y(a) \gamma_{1}=D_{*}^{\alpha} y(0)
$$

We can obtain the following first-order approximation by substitution of (6.9) in (6.8)

$$
y_{1}(x)=y_{0}(x)-\frac{(\alpha-1)}{2} J^{\alpha}\left[\sum_{k=0}^{\infty} P_{k} D_{*}^{\alpha} y(x)-g_{0}(x)-\lambda \int_{0}^{a} H(x, s) \tilde{y}_{0}(s) d s\right] .
$$

Finally, by substituting the constant values of $\gamma_{0}$ and $\gamma_{1}$ in (6.10) we have the results as the approximate solutions of (1.1)-(1.3), see the further details in [36-40].

\section{Applications}

In this section, we have applied homotopy perturbation method and variational iteration method to fractional Fredholm Integrodifferential equations with known exact solution.

Example 7.1. Consider the following linear Fredholm Integrodifferential equation:

$$
D^{\alpha} y(x)=\left(\frac{3}{2}+\frac{e^{2 x}}{2}\right)+\int_{0}^{x} e^{t} y(t) d t \quad 0 \leq x \leq 1,1<\alpha \leq 2
$$


Abstract and Applied Analysis

with initial boundary conditions

$$
y(0)=1, \quad y^{\prime}(1)=e
$$

the exact solution is $y(x)=e^{x}$. Now we construct

$$
D^{\alpha} y(x)=p\left(\left(\frac{3}{2}+\frac{e^{2 x}}{2}\right)+\int_{0}^{x} e^{t} y(t) d t\right) .
$$

Substitution of (5.4) in (7.3) and then equating the terms with same powers of $p$, we get the series

$$
\begin{aligned}
& p^{0}: D^{\alpha} y_{0}(x)=0 \\
& p^{1}: D^{\alpha} y_{1}(x)=\left(\frac{3}{2}+\frac{2}{3} e^{2 x}\right)+\int_{0}^{x} e^{t} y_{0}(t) d t \\
& p^{2}: D^{\alpha} y_{2}(x)=-\int_{0}^{x} e^{t} y_{1}(t) d t
\end{aligned}
$$

Now applying the operator $J_{\alpha}$ to the equations (7.4) and using initial-boundary conditions yields

$$
\begin{gathered}
y_{0}(x)=1 \\
y_{1}(x)=1+A x+J^{\alpha}\left(\left(\frac{3}{2}+\frac{e^{2 x}}{2}\right)+\int_{0}^{x} e^{t} y_{0} d t\right) \\
y_{2}(x)=J^{\alpha}\left(\int_{0}^{x} e^{t} y_{1} d t\right) \\
y_{n}(x)=J^{\alpha}\left(\int_{0}^{x} e^{t} y_{n-1} d t\right), \quad n=2,3,4, \ldots
\end{gathered}
$$

Then by solving (7.5)-(7.8), we obtain $y_{1}, y_{2}, \ldots$ as

$$
\begin{aligned}
y_{1}(x)= & 1+A x+\frac{5 x^{\alpha}}{2 \Gamma(\alpha+1)}+\frac{2 x^{\alpha+1}}{\Gamma(\alpha+2)}+\frac{3 x^{\alpha+2}}{2 \Gamma(\alpha+3)}+\frac{5 x^{\alpha+3}}{6 \Gamma(\alpha+4)} \\
y_{2}(x)= & \frac{x^{\alpha+1}}{\Gamma(\alpha+2)}+(A+1) \frac{x^{\alpha+2}}{2 \Gamma(\alpha+3)}+\left(\frac{A}{3}+\frac{1}{2}\right) \frac{x^{\alpha+3}}{\Gamma(\alpha+4)} \\
& +\left(\frac{A}{8}+\frac{1}{12}\right) \frac{x^{\alpha+4}}{\Gamma(\alpha+5)}+\frac{A x^{\alpha+5}}{15 \Gamma(\alpha+6)}+\frac{5 x^{2 \alpha+1}}{2 \Gamma(2 \alpha+2)}+\cdots
\end{aligned}
$$


Table 1: Values of $A$ for different values of $\alpha$.

\begin{tabular}{ccccc}
\hline & $\alpha=1.25$ & $\alpha=1.5$ & $\alpha=1.75$ & $\alpha=2$ \\
\hline$A$ & -2.33209843875457 & -1.906444021198994 & -0.88898224618462 & -0.098915873901025 \\
\hline
\end{tabular}

Table 2: Value of $A$ for different values of $\alpha$ using (7.14).

\begin{tabular}{ccccc}
\hline & $\alpha=1.25$ & $\alpha=1.5$ & $\alpha=1.75$ & $\alpha=2$ \\
\hline$A$ & 1.23429062479478 & 0.73267858113358 & 0.66218167845861 & 0.54744784230252 \\
\hline
\end{tabular}

Now, we can form the 2 term approximation as follows:

$$
\begin{aligned}
\phi_{2}(x)= & +A x+\frac{5 x^{\alpha}}{2 \Gamma(\alpha+1)}+\frac{3 x^{\alpha+1}}{\Gamma(\alpha+2)}+(A+2) \frac{x^{\alpha+2}}{2 \Gamma(\alpha+3)} \\
& +(A+4) \frac{x^{\alpha+3}}{3 \Gamma(\alpha+4)}+\left(\frac{A}{8}+\frac{1}{12}\right) \frac{x^{\alpha+4}}{\Gamma(\alpha+5)}+\frac{A x^{\alpha+5}}{15 \Gamma(\alpha+6)}+\frac{5 x^{2 \alpha+1}}{2 \Gamma(2 \alpha+2)}+\cdots,
\end{aligned}
$$

where $A$ can be determined by imposing initial-boundary conditions (7.2) on $\phi_{2}$. Table 1 shows the values of $A$ for different values of $\alpha$.

Now, we solve (7.1)-(7.2) by variational iteration method. According to variational iteration method, the formula (6.8) for (7.1) can be expressed in the following form:

$$
y_{k+1}(x)=y_{k}(x)-\frac{(\alpha-1)}{2} J^{\alpha}\left[D^{\alpha} y(x)-\left(\frac{3}{2}+\frac{e^{2 x}}{2}\right)-\int_{0}^{x} e^{t} y(t) d t\right]
$$

Then, in order to avoid the complex and difficult fractional integration, we can consider the truncated Taylor expansions for exponential term in (7.6)-(7.8) for example, $e^{x} \sim 1+x+$ $x^{2} / 2+x^{3} / 6$ and further, suppose that an initial approximation has the following form which satisfies the inial-boundary conditions

$$
y_{0}(x)=1+A x .
$$

Now by iteration formula (7.12), the first approximation takes the following form:

$$
\begin{aligned}
y_{1}(x)=y_{0}(x)-\frac{(\alpha-1)}{2} J^{\alpha}[ & \left.D^{\alpha} y_{0}(x)-\left(\frac{3}{2}+\frac{e^{2 x}}{2}\right)-\int_{0}^{x} e^{t} y_{0}(t) d t\right] \\
=1+A x+\frac{(\alpha-1)}{2} x^{\alpha} & {\left[\frac{5}{\Gamma(\alpha+1)}+\frac{2 x}{\Gamma(\alpha+2)}+\frac{(A+3) x^{2}}{2 \Gamma(\alpha+3)}\right.} \\
+ & \left.\frac{(5 / 2+A) x^{3}}{3 \Gamma(\alpha+4)}+\frac{(A / 2+1 / 6) x^{4}}{6 \Gamma(\alpha+5)}-\frac{A x^{5}}{30 \Gamma(\alpha+6)}\right] .
\end{aligned}
$$

By imposing initial-boundary conditions (7.2) on $y_{1}$, we can obtain the values of $A$ for different $\alpha$ which we show in Table 2. 
Example 7.2. Consider the following linear Fredholm Integrodifferential equation:

$$
D^{\alpha} y(x)=\left(1-\frac{x}{4}\right)+\int_{0}^{x} x t y^{2}(t) d t \quad 0 \leq x \leq 1,1<\alpha \leq 2
$$

with initial boundary conditions

$$
y(0)=0, \quad y^{\prime}(1)=1
$$

then the exact solution is $y(x)=x$. By applying the HPM, we have

$$
D^{\alpha} y(x)=p\left(\left(1-\frac{x}{4}\right)+\int_{0}^{x} x t y^{2}(t) d t\right) .
$$

Substitution of (5.4) in (7.15) and then equating the terms with same powers of $p$, we get the following series expressions:

$$
\begin{aligned}
& p^{0}: D^{\alpha} y_{0}(x)=0 \\
& p^{1}: D^{\alpha} y_{1}(x)=\left(1-\frac{x}{4}\right)+\int_{0}^{x} x t y_{0}^{2}(t) d t \\
& p^{2}: D^{\alpha} y_{2}(x)=2 \int_{0}^{x} x t y_{0}(t) y_{1}(t) d t \\
& p^{3}: D^{\alpha} y_{3}(x)=\int_{0}^{x} x t\left(y_{0}(t) y_{2}(t)+y_{1}^{2}(t)\right) d t \\
& p^{4}: D^{\alpha} y_{4}(x)=\int_{0}^{x} x t\left(2 y_{0}(t) y_{4}(t)+2 y_{1} y_{3}+y_{2}{ }^{2}(t)\right) d t
\end{aligned}
$$

Applying the operator $J_{\alpha}$ to (7.18) and using initial-boundary conditions, then we get

$$
\begin{aligned}
& y_{0}(x)=0, \\
& y_{1}(x)=A x+J^{\alpha}\left(\left(1-\frac{x}{4}\right)+\int_{0}^{x} x t y_{0}^{2}(t) d t\right), \\
& y_{2}(x)=0, \\
& y_{3}(x)=J^{\alpha}\left(\int_{0}^{x} x t\left(y_{0}(t) y_{2}(t)+y_{1}^{2}(t)\right) d t\right), \\
& y_{4}(x)=J^{\alpha}\left(\int_{0}^{x} x t\left(2 y_{0}(t) y_{4}(t)+2 y_{1} y_{3}+y_{2}^{2}(t)\right) d t\right),
\end{aligned}
$$


Table 3: Value of $A$ for different values of $\alpha$.

\begin{tabular}{ccccc}
\hline & $\alpha=1.25$ & $\alpha=1.5$ & $\alpha=1.75$ & $\alpha=2$ \\
\hline$A$ & 0.179304 & 0.153796 & 0.0673477 & 0.124989 \\
\hline
\end{tabular}

Table 4: Value of $A$ for different values of $\alpha$.

\begin{tabular}{ccccc}
\hline & $\alpha=1.25$ & $\alpha=1.5$ & $\alpha=1.75$ & $\alpha=2$ \\
\hline$A$ & 0.88967375 & 0.76492075 & 0.650263 & 0.5625 \\
\hline
\end{tabular}

Thus, by solving (7.19), we obtain $y_{1}, y_{2}, y_{3}, \ldots$

$$
\begin{gathered}
y_{1}(x)=A x+\frac{x^{\alpha}}{\Gamma(\alpha+1)}-\frac{x^{\alpha+1}}{4 \Gamma(\alpha+2)}, \\
y_{2}(x)=0, \\
y_{3}(x)=\frac{A^{2} x^{\alpha+5}}{4 \Gamma(\alpha+6)}+\frac{2 A x^{2 \alpha+4}}{(\alpha+3) \Gamma(\alpha+1) \Gamma(2 \alpha+5)}+\frac{x^{3 \alpha+3}}{(2 \alpha+2) \Gamma(\alpha+1) \Gamma(\alpha+1) \Gamma(3 \alpha+4)}+\cdots .
\end{gathered}
$$

Now, we can form the 3 term approximation

$$
\begin{aligned}
\phi_{2}(x)= & A x+\frac{x^{\alpha}}{\Gamma(\alpha+1)}-\frac{x^{\alpha+1}}{4 \Gamma(\alpha+2)}+\frac{A^{2} x^{\alpha+5}}{4 \Gamma(\alpha+6)}+\frac{2 A x^{2 \alpha+4}}{(\alpha+3) \Gamma(\alpha+1) \Gamma(2 \alpha+5)} \\
& +\frac{x^{3 \alpha+3}}{(2 \alpha+2) \Gamma(\alpha+1) \Gamma(\alpha+1) \Gamma(3 \alpha+4)}+\cdots,
\end{aligned}
$$

where $A$ can be determined by imposing initial-boundary conditions (7.16) on $\phi_{2}$. Thus, we have Table 3.

Similarly, by variational iteration method we have the following form:

$$
y_{k+1}(x)=y_{k}(x)-\frac{(\alpha-1)}{2} J^{\alpha}\left[D^{\alpha} y(x)-\left(1-\frac{x}{4}\right)+\int_{0}^{x} x t y^{2}(t) d t\right]
$$

where we suppose that an initial approximation has the following form which satisfies the initial-boundary conditions $y_{0}(x)=A x$. Now by using the iteration formula, the first approximation takes the following form:

$$
\begin{aligned}
y_{1}(x) & =y_{0}(x)-\frac{(\alpha-1)}{2} J^{\alpha}\left[D^{\alpha} y_{0}(x)-\left(1-\frac{x}{4}\right)+\int_{0}^{x} x t y_{0}^{2}(t) d t\right] \\
& =A x+\frac{(\alpha-1)}{2} J^{\alpha}\left[\frac{-x^{\alpha}}{\Gamma(\alpha+1)}+\frac{x^{\alpha+1}}{4 \Gamma(\alpha+2)}\right] .
\end{aligned}
$$

By imposing initial-boundary conditions, we can obtain the following Table 4 . 


\section{Conclusion}

In this work, homotopy perturbation method (HPM) and variational iteration method (VIM) have been applied to linear and nonlinear initial-boundary value problems for fractional Fredholm Integrodifferential equations. Two examples are presented in order to illustrate the accuracy of the present methods. Comparisons of HPM and VIM with exact solution have been given in the Tables 1-4.

\section{Acknowledgment}

The authors would like to thank the referee(s) for the very constructive comments and valuable suggestions including attention to $[12,17-26]$ that improved the paper very much.

\section{References}

[1] A. A. Kilbas, H. M. Srivastava, and J. J. Trujillo, Theory and Applications of Fractional Differential Equations, vol. 204 of North-Holland Mathematics Studies, Elsevier Science B.V., Amsterdam, The Netherlands, 2006.

[2] V. Lakshmikantham, S. Leela, and J. V. Devi, Theory of Fractional Dynamic Systems, Cambridge Scientific, 2009.

[3] K. S. Miller and B. Ross, An Introduction to the Fractional Calculus and Fractional Differential Equations, A Wiley-Interscience Publication, John Wiley \& Sons, New York, NY, USA, 1993.

[4] I. Podlubny, Fractional Differential Equations, vol. 198 of Mathematics in Science and Engineering, Academic Press, San Diego, Calif, USA, 1999.

[5] K. Diethelm and A. D. Freed, "On the solution of nonlinear fractional order differential equations used in the modeling of viscoelasticity," in Scientific Computing in Chemical Engineering II-Computational Fluid Dynamics, Reaction Engineering and Molecular Properties, F. Keil, W. Mackens, H. Voss, and J. Werther, Eds., pp. 217-224, Springer, Heidelberg, Germany, 1999.

[6] R. Metzler, W. Schick, H.-G. Kilian, and T. F. Nonnenmacher, "Relaxation in filled polymers: a fractional calculus approach," Journal of Chemical Physics, vol. 103, no. 16, pp. 7180-7186, 1995.

[7] L. Gaul, P. Klein, and S. Kemple, "Damping description involving fractional operators," Mechanical Systems and Signal Processing, vol. 5, no. 2, pp. 81-88, 1991.

[8] W. G. Glockle and T. F. Nonnenmacher, "A fractional calculus approach of self-similar protein dynamics," Biophysical Journal, vol. 68, pp. 46-53, 1995.

[9] R. Hilfert, Applications of Fractional Calculus in Physics, World Scientific, River Edge, NJ, USA, 2000.

[10] R. P. Agarwal, M. Benchohra, and S. Hamani, "A survey on existence results for boundary value problems of nonlinear fractional differential equations and inclusions," Acta Applicandae Mathematicae, vol. 109, no. 3, pp. 973-1033, 2010.

[11] Z. Bai and H. Lü, "Positive solutions for boundary value problem of nonlinear fractional differential equation," Journal of Mathematical Analysis and Applications, vol. 311, no. 2, pp. 495-505, 2005.

[12] D. Baleanu, K. Diethelm, E. Scalas, and J. J. Trujillo, Fractional Calculus, vol. 3 of Series on Complexity, Nonlinearity and Chaos, World Scientific, Hackensack, NJ, USA, 2012.

[13] P. K. Kythe and P. Puri, Computational Methods for Linear Integral Equations, Birkhäuser, Boston, Mass, USA, 2002.

[14] Z.-B. Li and J.-H. He, "Fractional complex transform for fractional differential equations," Mathematical \& Computational Applications, vol. 15, no. 5, pp. 970-973, 2010.

[15] F. Mainardi, "Fractional calculus: some basic problems in continuum and statistical mechanics," in Fractals and Fractional Calculus in Continuum Mechanics, vol. 378 of CISM Courses and Lectures, pp. 291348, Springer, Vienna, Austria, 1997.

[16] A.-M. Wazwaz, "A comparison study between the modified decomposition method and the traditional methods for solving nonlinear integral equations," Applied Mathematics and Computation, vol. 181, no. 2, pp. 1703-1712, 2006.

[17] J.-H. He, "Variational iteration method-a kind of non-linear analytical technique: some examples," International Journal of Non-Linear Mechanics, vol. 34, no. 4, pp. 699-708, 1999. 
[18] J.-H. He, "Homotopy perturbation method: a new nonlinear analytical technique," Applied Mathematics and Computation, vol. 135, no. 1, pp. 73-79, 2003.

[19] J.-H. He, "Homotopy perturbation technique," Computer Methods in Applied Mechanics and Engineering, vol. 178, no. 3-4, pp. 257-262, 1999.

[20] J.-H. He, "A coupling method of a homotopy technique and a perturbation technique for non-linear problems," International Journal of Non-Linear Mechanics, vol. 35, no. 1, pp. 37-43, 2000.

[21] J. H. He, Non-Pertubation methods for strongly nonlinear problems [dissertation], Internet Gmbh, Berlin, Germany, 2006.

[22] J.-H. He, "Approximate analytical solution for seepage flow with fractional derivatives in porous media," Computer Methods in Applied Mechanics and Engineering, vol. 167, no. 1-2, pp. 57-68, 1998.

[23] J.-H. He, "A short remark on fractional variational iteration method," Physics Letters A, vol. 375, no. 38, pp. 3362-3364, 2011.

[24] J.-H. He, "Homotopy perturbation method with an auxiliary term," Abstract and Applied Analysis, vol. 2012, Article ID 857612, 7 pages, 2012.

[25] H. E. Ji-Huan, "A Note on the homotopy perturbation method," Thermal Science, vol. 14, no. 2, pp. $565-568,2010$.

[26] J.-H. He, S. K. Elagan, and Z. B. Li, "Geometrical explanation of the fractional complex transform and derivative chain rule for fractional calculus," Physics Letters A, vol. 376, no. 4, pp. 257-259, 2012.

[27] S. Abbasbandy, "An approximation solution of a nonlinear equation with Riemann-Liouville's fractional derivatives by He's variational iteration method," Journal of Computational and Applied Mathematics, vol. 207, no. 1, pp. 53-58, 2007.

[28] O. Abdulaziz, I. Hashim, and S. Momani, "Application of homotopy-perturbation method to fractional IVPs," Journal of Computational and Applied Mathematics, vol. 216, no. 2, pp. 574-584, 2008.

[29] A. Le Méhauté, L. Nivanen, and A. El Kaabouchi, "Contribution of Non Integer Integro-differential operators (NIDO) to the geometrical understanding of Riemann's conjecture-(I)," in Proceedings of the 2nd IFAC Workshop on Fractional Differentiation and Its Applications (FDA '06), pp. 230-233, 2006.

[30] B. Ross, Fractional Calculus and Its Applications, vol. 457 of Lecture Notes in Mathematics, Springer, Berlin, Germany, 1975.

[31] U. Sumita, "The matrix Laguerre transform," Applied Mathematics and Computation, vol. 15, no. 1, pp. $1-28,1984$.

[32] A. Kilicman and Z. A. A. Al Zhour, "Kronecker operational matrices for fractional calculus and some applications," Applied Mathematics and Computation, vol. 187, no. 1, pp. 250-265, 2007.

[33] S. J. Liao, "An approximate solution technique not depending on small parameters: a special example," International Journal of Non-Linear Mechanics, vol. 30, no. 3, pp. 371-380, 1995.

[34] S.-J. Liao, "Boundary element method for general nonlinear differential operators," Engineering Analysis with Boundary Elements, vol. 20, no. 2, pp. 91-99, 1997.

[35] A. Yildırım, "Solution of BVPs for fourth-order integro-differential equations by using homotopy perturbation method," Computers E Mathematics with Applications, vol. 56, no. 12, pp. 3175-3180, 2008.

[36] H. Jafari, A. Kadem, D. Baleanu, and T. Yilmaz, "Solutions of the fractional Davey-Stewartson Equations with variational iteration method," Romanian Reports in Physics, vol. 64, no. 2, pp. 337-346, 2012.

[37] A. Kadem and A. Kiliçman, "The approximate solution of fractional Fredholm integrodifferential equations by variational iteration and homotopy perturbation methods," Abstract and Applied Analysis, vol. 2012, Article ID 486193, 10 pages, 2012.

[38] Y. Nawaz, "Variational iteration method and homotopy perturbation method for fourth-order fractional integro-differential equations," Computers $\mathcal{E}$ Mathematics with Applications, vol. 61, no. 8, pp. 2330-2341, 2011.

[39] Z. Odibat and S. Momani, "The variational iteration method: an efficient scheme for handling fractional partial differential equations in fluid mechanics," Computers $\mathcal{E}$ Mathematics with Applications, vol. 58, no. 11-12, pp. 2199-2208, 2009.

[40] N. H. Sweilam, "Fourth order integro-differential equations using variational iteration method," Computers \& Mathematics with Applications, vol. 54, no. 7-8, pp. 1086-1091, 2007. 


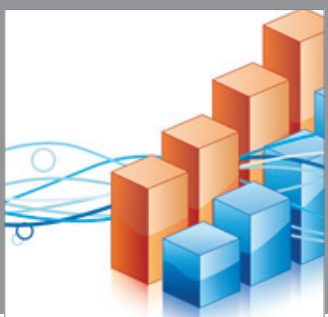

Advances in

Operations Research

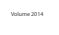

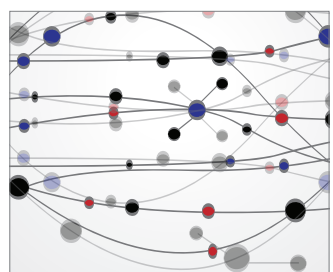

\section{The Scientific} World Journal
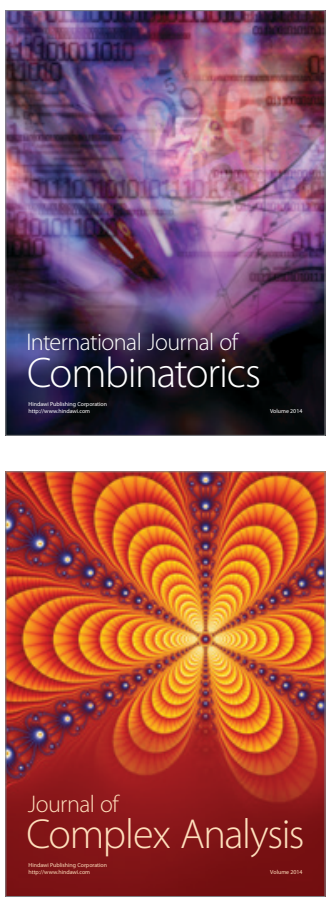

International Journal of

Mathematics and

Mathematical

Sciences
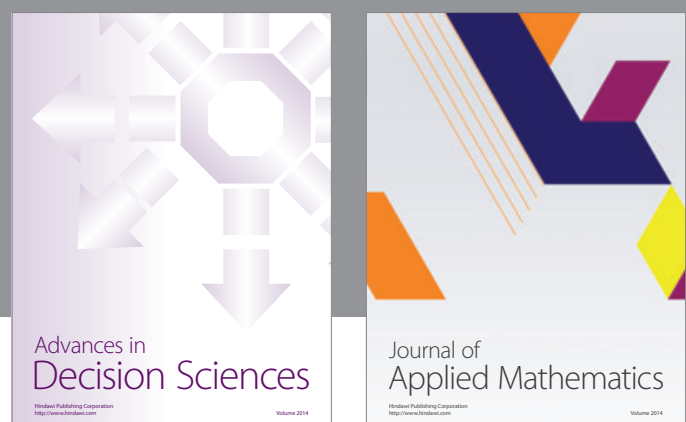

Journal of

Applied Mathematics
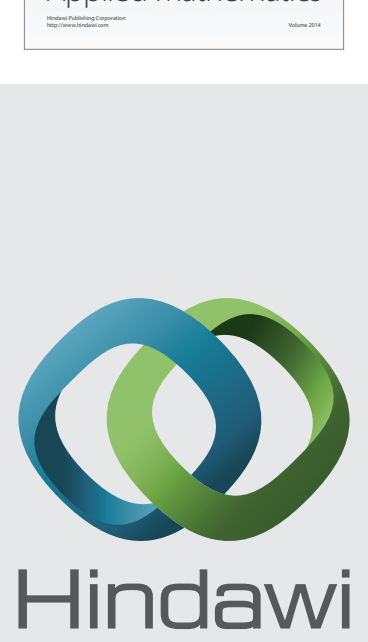

Submit your manuscripts at http://www.hindawi.com
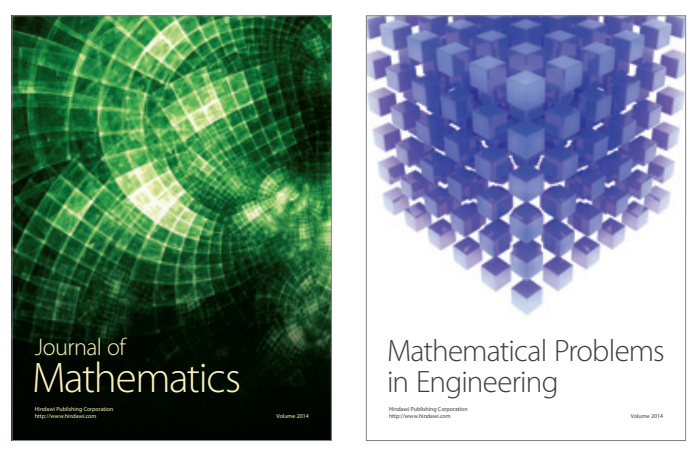

Mathematical Problems in Engineering
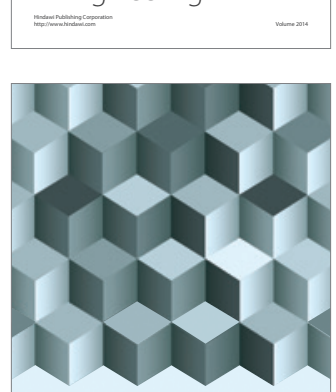

Journal of

Function Spaces
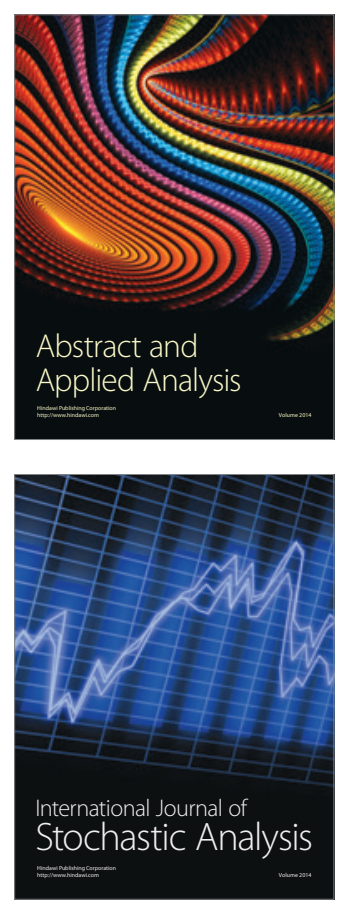

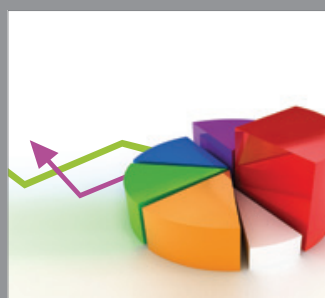

ournal of

Probability and Statistics

Promensencen
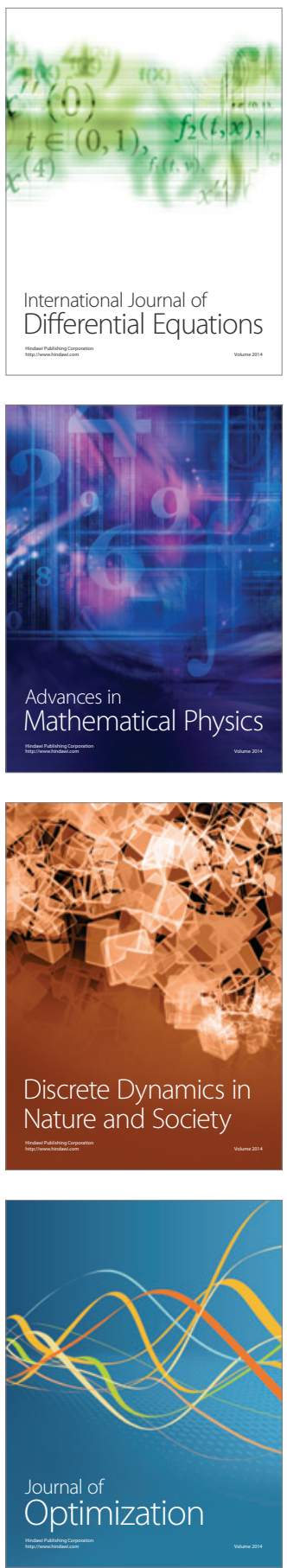